\title{
Enhanced optical properties of perovskite thin film through material optimization for photovoltaic application.
}

\author{
${ }^{*}$ SA Olaleru ${ }^{1,3,4}$, JK Kirui ${ }^{1}$, D Wamwangi ${ }^{2}$, L Jhamba ${ }^{1}$, R Erasmus ${ }^{2}$, B Mwakikunga ${ }^{4}$, *K \\ Roro $^{3}$ \\ 1. Physics Department, University of Venda, Thohoyandou, Limpopo 0950, South Africa \\ 2. Physics Department, University of Witwatersrand, Johannesburg 2050, South Africa \\ 3. CSIR-Energy Centre, Council for Scientific and Industrial Research, P.O. Box 395, \\ Pretoria 0001, South Africa \\ 4. DST/CSIR- National Centre for Nano-Structured Materials, P.O. Box 395, Pretoria \\ 0001, South Africa \\ *Corresponding authors: akinolaleru@gmail.com, kroro@csir.co.za
}

\begin{abstract}
The optical performance of the perovskite materials is enhanced through material optimization. This work seeks to establish the role of antisolvent and additive with new material composition on light absorption property. Due to this composition we extended the wavelengths to near Infrared range which is a suitable property for photovoltaic device. From the features of the film, optical parameters, together with anti-stoke shift and dielectric constant were calculated using Cauchy dispersion formalism. Based on our results, dielectric constant which is considered as a design parameter for photovoltaic cell and an unusual anti-stoke shift were observed. In sum, the optical properties are tied to material composition, morphology and technique used.
\end{abstract}

Key words: Perovskite, photovoltaic, antisolvent, optical properties

\section{Introduction}

Optical properties are among the most intriguing and beneficial tunable parameters of nanomaterials. This is based on the potential coupling of optical properties with different material properties and functionalities (for example electronic, magnetic and thermal) that are fundamentally essential to numerous innovative applications like solar cell, sensor and other optoelectronic devices.

As of late, hybrid perovskite materials have shown incredible possibilities for solar energy conversion and optoelectronics technologies by virtue of its benefits of high conversion efficiency, low-cost preparation and the application of earth-abundant materials, which are basic determinants for massive production. The optical properties of lead halide perovskites are of basic significance for almost all applications. Based on the screening from literature, the greater part of reports centre on the fabrication of photovoltaic devices, $[1,2]$ while photophysical processes of these materials are missing. A greater comprehension of the basic photo physics of perovskites will not only help device fabrication but equally produce emerging forms for these novel materials and at the same time generate guidelines for dealing with existing challenges. 
The quality of the film is the most critical determinant for establishing the quantification of optical properties of the perovskite film. The reasonable path to obtain high-quality perovskite film is careful monitoring of the grain size of perovskite crystal [3]. The introduction of antisolvent during the spin-coating process produced perovskite of intermediate phase. The role of antisolvent is to control the morphology of the perovskite material by producing rapid precipitation of the perovskite, giving rise to thick and homogeneous perovskite films. It has been recorded by some researchers that it is challenging to obtain a high-quality perovskite film by employing diethyl ether, chlorobenzene, or toluene as the antisolvent in damp environment [6]. More so, N,Ndimethylformamide (DMF) and dimethyl sulphoxide (DMSO) are frequently utilized as a mixing solvent for MAPbI3 precursors because of their synergetic impact. DMF is a solid polar solvent appropriate for dissolving MAI and PbI2, whereas DMSO has well established coordination impact with $\mathrm{Pb}^{2+}$ that can delay the formation of $\mathrm{PbI} 2$ and increase the quality of the perovskite films $[4,5,28]$. Watson et al., described ethyl acetate as an antisolvent employed for the development of pinhole-free MAPbI3 perovskite films in air ambience, where ethyl acetate serves as a desiccating agent, preserving the susceptible perovskite transitional period from water molecules in the course of deposition and annealing [6]. In short, antisolvent has hydrophobic properties that increase the life expectancy of the active layer. But, to the best of our knowledge, there are no information provided for the impact of antisolvent and additive variation on the optical properties of the perovskite film. In addition, most of the reported high-quality perovskite films were obtained with temperature over $100{ }^{\circ} \mathrm{C}$ [28-30]. In this context, high crystallized perovskite films were produced by annealing the films at $70{ }^{\circ} \mathrm{C}$ for $10 \mathrm{~min}$. Accordingly, evolvement of regulated crystallization protocol that is suitable with low-temperature deposition of precursor films is greatly required for mass scale production of perovskite thin film.

In this work, we used DMSO as an additive in DMF to regulate the crystal growth by dissolving the residue of $\mathrm{PbI} 2$ which can impede the crystallization and ethyl acetate as an anti-solvent to control the morphology of the perovskite film resulting in improved homogeneity. Their impacts on optical properties were examined along with consequent improvement on the light absorption property. As a follow up, we sought to investigate how they relate to dielectric constant and stoke shift.

\section{Experimental section}

\subsection{Materials and method}

The organic source is Methylammonium iodide $(\mathrm{CH} 3 \mathrm{NH} 3 \mathrm{I})$ and the inorganic source is lead iodide ( $\mathrm{PbI} 2)$. Most of the materials used were bought from Sigma Aldrich. Deposition of perovskite layer was done by a single-step spin-coating method followed by direct annealing.

CH3NH3I $(0.160 \mathrm{~g})$ and PbI2 (0.460g) were dissolved in solvent ratio of 1:4 (DMSO: DMF). The solution was heated on a hotplate with stirring for about $20 \mathrm{~min}$ at $70{ }^{\circ} \mathrm{C}$ for complete reaction. $\mathrm{CH} 3 \mathrm{NH} 3 \mathrm{I}$ and $\mathrm{PbI} 2$ in a molar ratio of 1:1 were dispersed on the substrate using a spin coater. For spin coating method, the $0.1 \mathrm{~mL}$ solution was spun at $2500 \mathrm{rpm}$ for $30 \mathrm{~s}$, while $0.2 \mathrm{~mL}$ ethyl acetate was spun across the film during spinning at $2500 \mathrm{rpm}$ in $10 \mathrm{~s}$ after the spin-coating began. To obtain the crystallization of the perovskite layer, the film was annealed at $70{ }^{\circ} \mathrm{C}$ on a hotplate for about $10 \mathrm{~min}$ and then cooled at room temperature as shown in Figure 1. The pristine perovskite materials were prepared using similar method in the absence of DMSO as an additive. The quality and surface morphology of the perovskite layer depends on the concentration of the precursor solution, the speed at which the substrate is rotated during spin coating and the annealing process parameters, such as annealing time and temperature for crystallization. 


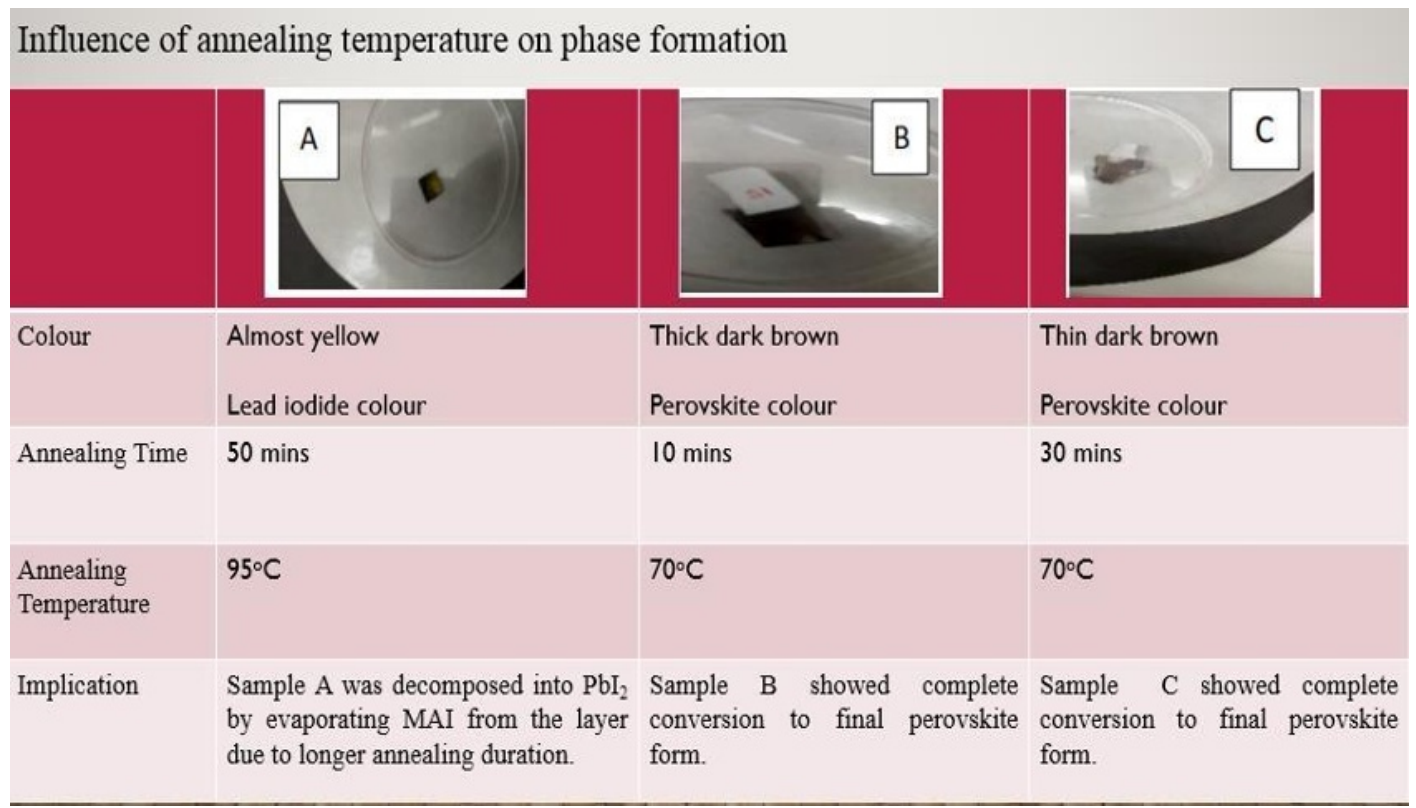

Figure 1: Influence of annealing temperature on phase formation

The formation of perovskite structure requires adequate heat treatment whose purpose is twofold; removal of excess solvent remaining on the film and speeding up crystallization of perovskite structure. Therefore, based on our results in Figure 1 it was necessary to keep annealing temperature at $70{ }^{\circ} \mathrm{C}$ to avoid $\mathrm{PbI}_{2}$ formation and annealing time should be kept below 30 mins.

\subsection{Characterization instruments (Structural and Optical Measurements)}

The XRD pattern was recorded using an X-ray diffractometer with $\mathrm{Cu}-\mathrm{k} \alpha$ radiation (XPert Pro X-Ray Diffractometer Panalytical Ltd, Eindhoven, Netherlands). Optical absorption measurements were carried out in the wavelength range (200-1400 nm) using a UV-Visible spectrophotometer. The photoluminescence (PL) and excitation spectra (PLE) were recorded using fluorolog-3 spectrophotometer with double-grating in both excitation and emission monochromators. The morphology of the films was analysed by scanning electron microscope equipment SEM (Zeiss, Berlin, Germany). The film thickness and roughness were determined by Atomic Force Microscopes (AFM) Veeco Multimode.

\section{Results and discussions}

\subsection{Structural and grain size}

Figure 2 indicates the X-ray diffraction pattern of perovskite film prepared on FTO glass substrate. The main diffraction peaks located at $14.1^{\circ}, 28.4^{\circ}, 31.9^{\circ}, 40.6^{\circ}$ and $43.2^{\circ}$ for the $2 \Theta$ scan are seen, related to the planes of (110), (220), (222), (224) and (314) of perovskite, which are similar with XRD pattern in ref $[7,8,26]$. Based on the crystal planes obtained we can ascertain that the reaction of MAI with $\mathrm{PbI}_{2}$ was complete because the peaks correlated with $\mathrm{PbI}_{2}$ and MAI were not noticed and the perovskite film is highly crystalline perovskite phase. 
The grain size was evaluated to be $30 \mathrm{~nm}$ based on the Debye-Scherrer formula regarding as calculation technique in $[9,10]$ i.e. $\mathrm{D}=\mathrm{K} \lambda / \beta \cos \Theta$, where $\mathrm{D}$ stand for the grain size, $\mathrm{k}$ is constant (0.91). $\lambda$ is the wavelength of the incident X-ray $(0.154 \mathrm{~nm}), \beta$ is identified as the full width at half maximum of the XRD peak in radian unit and $\Theta$ is the diffraction angle, with $2 \Theta$ of $14.1^{\circ}$ taken for our Grain size determination. Figure 3 reveals the surface topography of the $\mathrm{MAPbI}_{3}$ based perovskite film by AFM measured at a resolution of $30 \mathrm{x}$ $30 \mu \mathrm{m}^{2}$ to yield rms roughness of $7.7 \mathrm{~nm}$. The average height to height correlations agree with the morphology image of Figure 4 for the $\mathrm{MAPbI}_{3}$ perovskite film synthesized using DMSO additive in the solvent. We concluded that indeed the use of ethyl acetate as an antisolvent resulted in crystals that were uniformly formed with less pinholes. A reasonably high crystallinity in which the size distribution is partially homogeneous is observed.

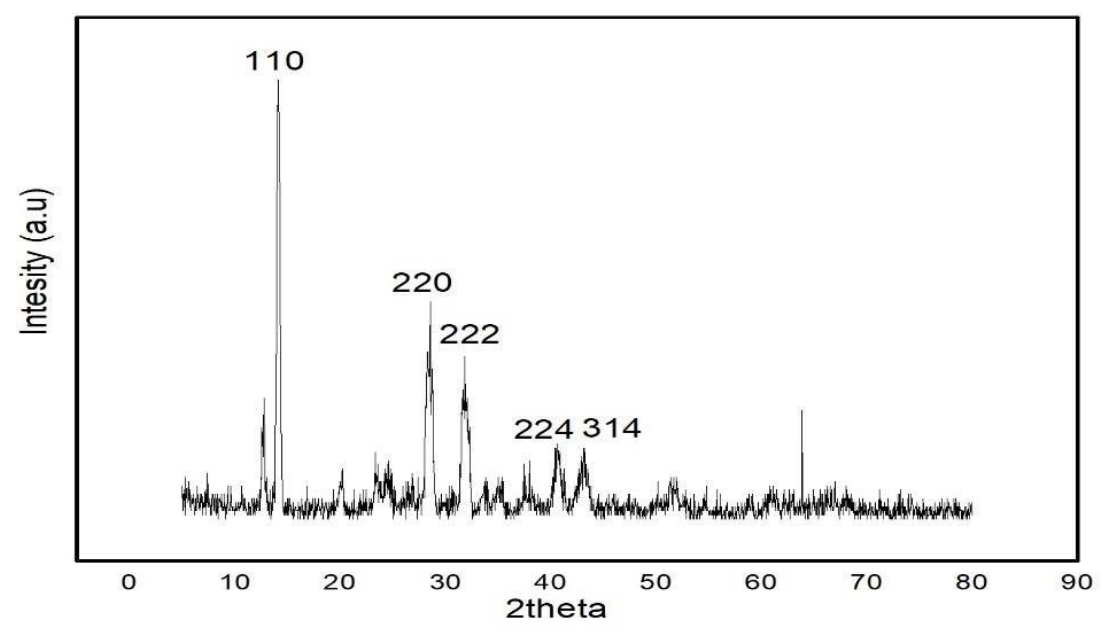

Figure 2. XRD pattern of perovskite film on FTO glass substrate.

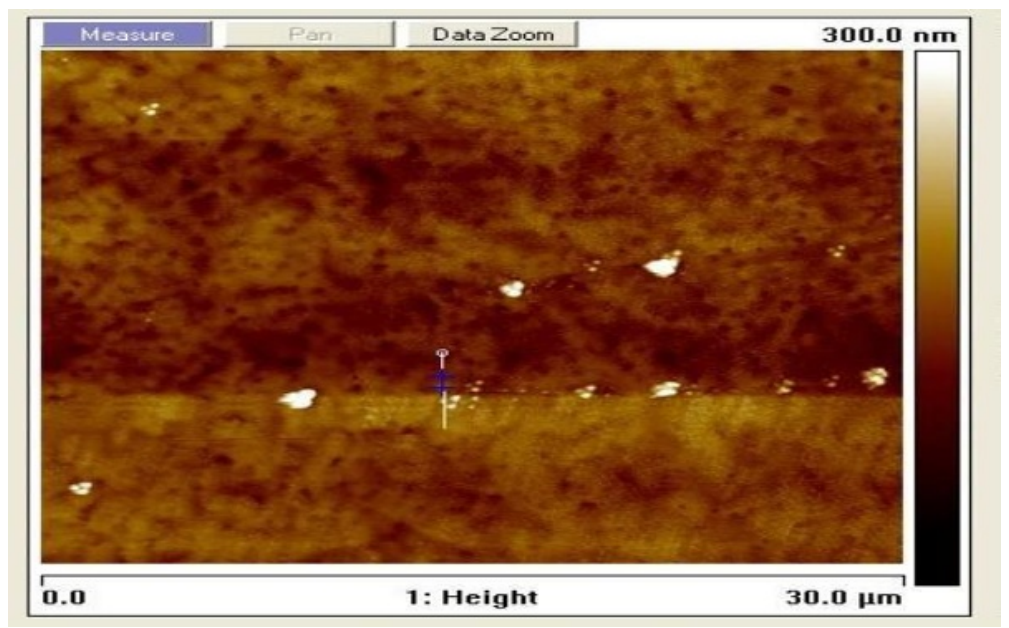

Figure 3. AFM image of the perovskite layer 


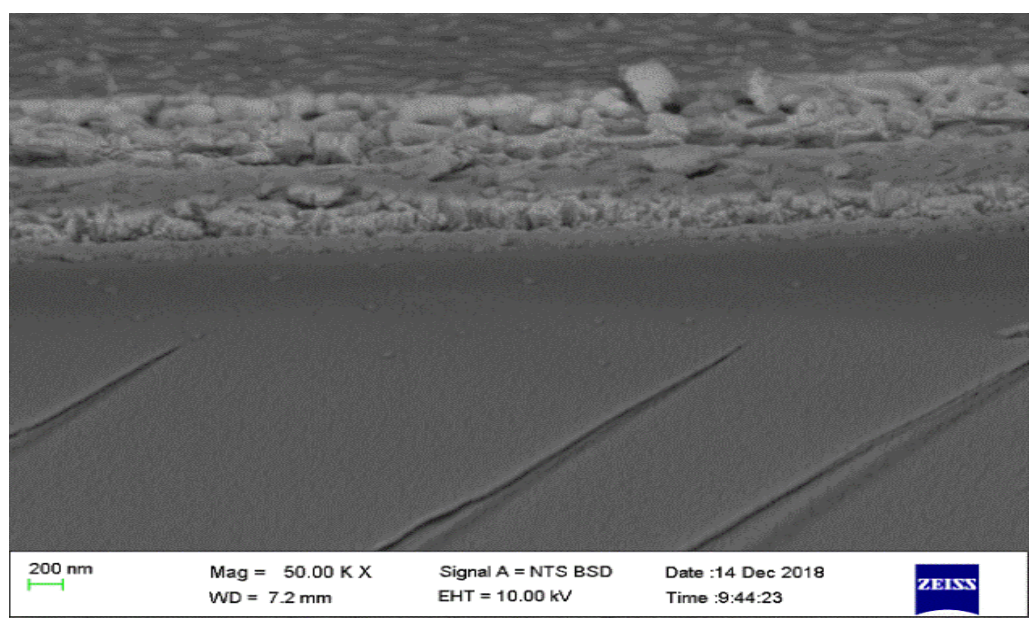

Figure 4. SEM images of the perovskite layer

\subsection{Optical analysis}

The UV-Vis optical absorption and $\mathrm{PL}$ spectra of $\mathrm{CH}_{3} \mathrm{NH}_{3} \mathrm{PbI}_{3}$ perovskite thin film were investigated using spectrophotometers at room temperature.

\subsubsection{Absorption and Emission Spectra}

The failure to absorb infrared (IR) light in the wavelength ranges, which form half of the energy of the whole solar spectrum as shown in Figure 5, constitutes a huge energy loss by traditional solar cells and Dye-sensitized solar cells. In this work, through material optimization we extended the response wavelength to the near IR range.
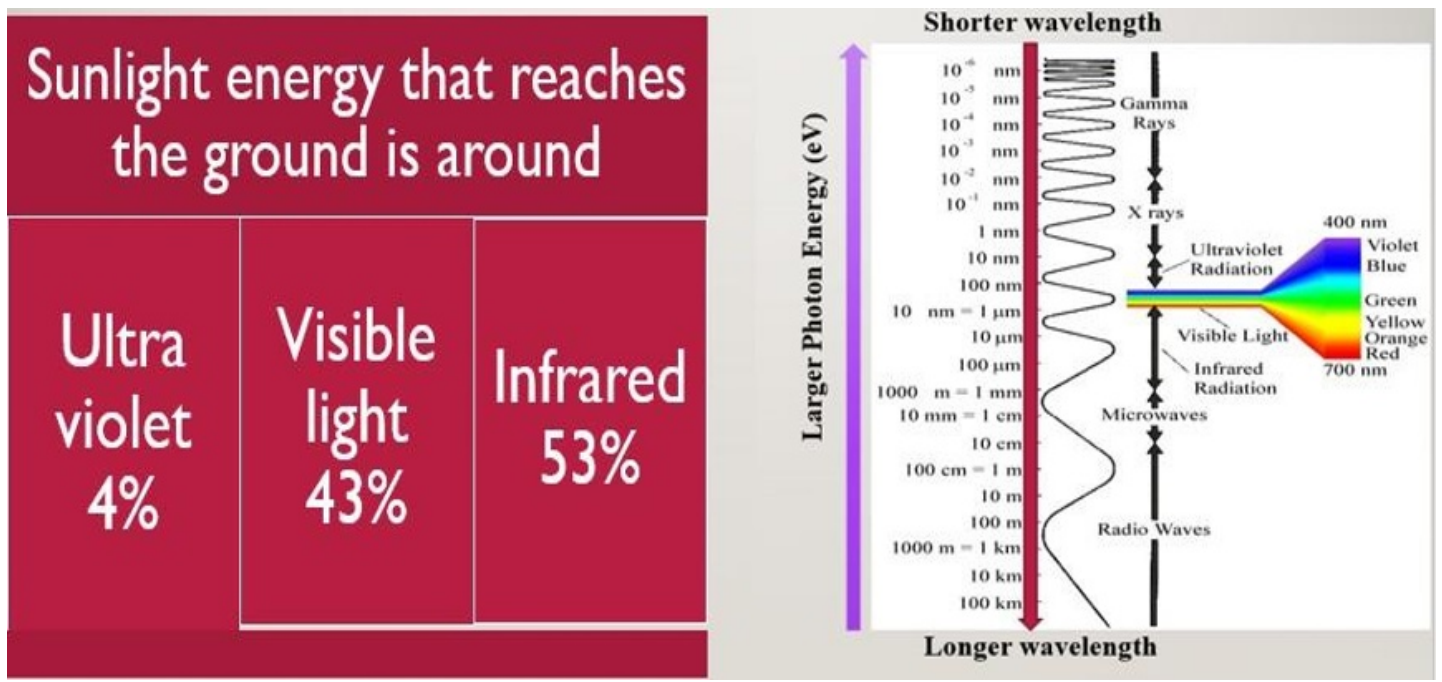

Figure 5: Solar emission spectrum

Ultraviolet/visible (UV-Vis) absorption spectrum was measured in the wavelength region of 200-1400 nm with spectrophotometer as illustrated in Figure 6. The absorption edge is 
located at around 797.50nm, which corresponds to the optical band gap $(1.55 \mathrm{eV})$. The perovskite layers show that their absorption is in the red near IR region and it can be tuned to match the solar emission spectrum. The sharp edge of the absorption of $\mathrm{MAPbI}_{3}$ shown in Figure 6 is an indication for low defect density and step- like absorption like that is needful for better solar cells as required by the detailed-balance theory [20,21]. More so, the absorption here goes beyond $700 \mathrm{~nm}$ and it is a suitable property for optoelectronic devices. There is no significant difference between the two UV-Vis spectra except for absorption edge of perovskite-DMSO that is slightly lower than pristine.

There is an absorption edge around $600 \mathrm{~nm}$ although not as sharp as the one around 800 $\mathrm{nm}$, this further unveil unexplored defects/traps. It was also observed that two absorption peaks emerged in the infrared - one around $900 \mathrm{~nm}$ and the other around $1200 \mathrm{~nm}$. This implies that absorption range of lead halide perovskite is not restricted to the visible region as reported in some literatures [32]. But, by further optimizing the morphology and composition of perovskite materials, the near infrared (NIR) photo response of PSCs can be enhanced.

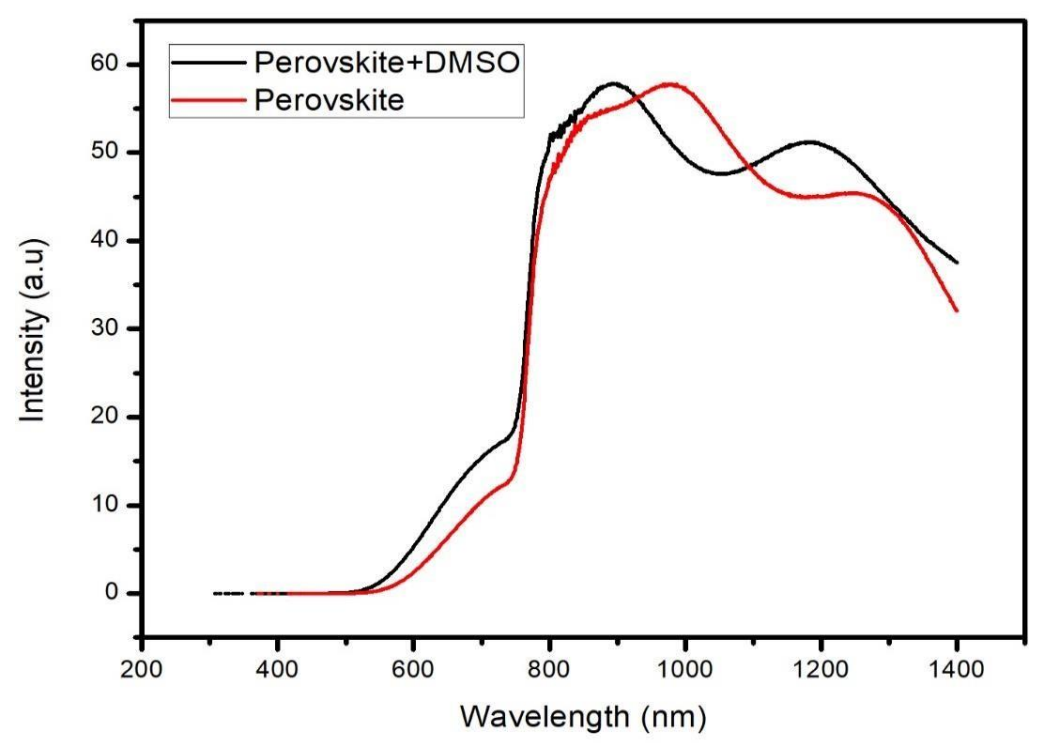

Figure 6. UV/Vis absorption spectra for pristine perovskite and perovskite-DMSO thin film.

The features from photoluminescence (PL) spectrum provide crucial information about various material properties like crystallinity, quantum efficiency, presence of trap and defect state, recombination mechanism and even phase transformation. All these information may be deduced from the intensity, linewidth and special content of the PL spectra as shown in Figure 7. 


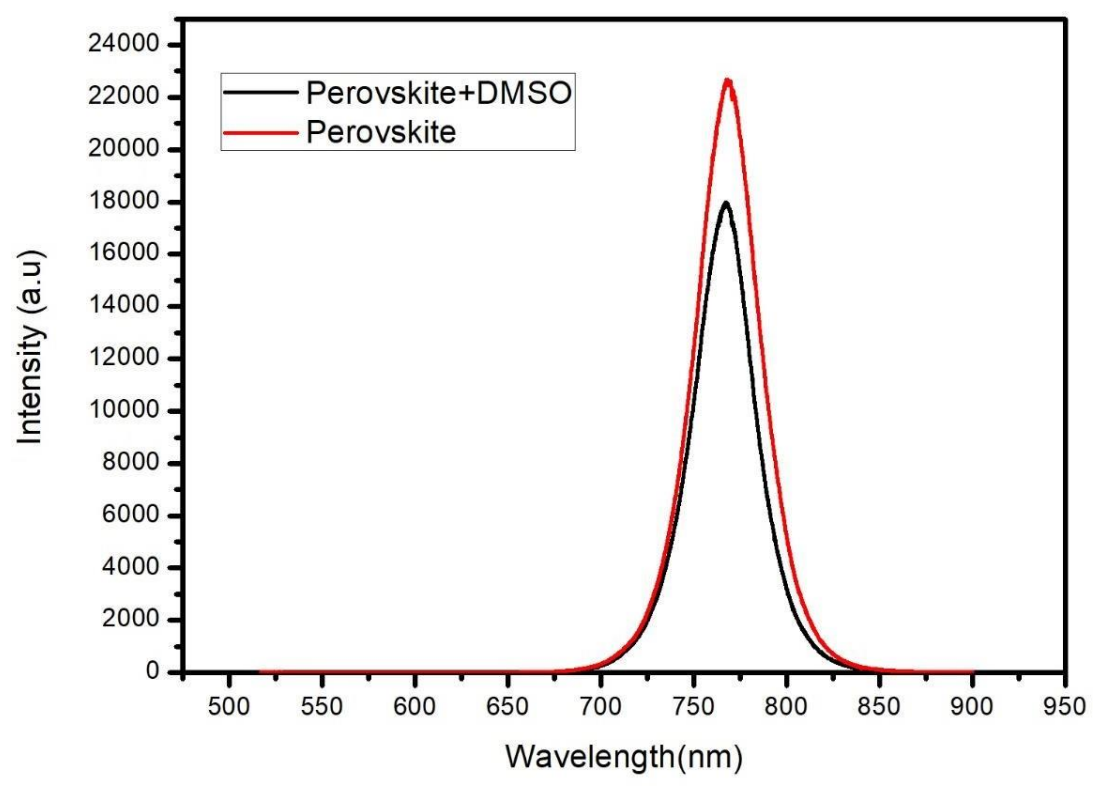

Figure 7. PL spectra for pristine perovskite and perovskite-DMSO thin film

The criterion for describing the optical quality of a material through photoluminescence measurement is the width of the emission band as shown in Figure 7. When the width of the PL peak that usually determined as of full width at half maximum (FWHM) shows smaller values, this implies better structural uniformity such as width of several quantum wells, composition uniformity. The PL spectrum of pristine material shows strong peak while the PL spectrum of perovskite-DMSO film drop which may be attributed to viscosity and defect states that leads to a non-radiative recombination.

It can be observed that the thin film for the PL properties, being excited by a $514.5 \mathrm{~nm}$, show a typical luminescence behaviour with the emission of a dominant intense peak centred at around $769.79 \mathrm{~nm}$. These perovskite materials exhibit band to band emission at room temperature. The film with smaller crystallite could shows higher luminescence intensity compared to larger crystallites. This is in conformity with crystallite size got from Debye-Scherrer formula. We do not observe any quantum confinement effect because the crystalline domain (as specified by SEM and XRD) are bigger than the recorded Bohr radius $\left(2.2 \mathrm{~nm}\right.$ for $\left.\mathrm{MAPbI}_{3}\right)$ [27].

Two observations can be made from the spectrum. First, the strong peak intensity is indicative of radiative recombination (Wannier-Mott exciton). Second, the transition energy is red shifted by $0.16 \mathrm{eV}$, which can be as well regarded as a desired behaviour, since the emission (absorption) at longer wavelengths is an important researcher's aim.

Emission band red shifted from the absorption edge is seen which is generally assigned to defect states [22] viz; vacancies, imperfections. Typically, red-shifted emission peak is caused by spontaneous radiative recombination within such trapped states in contrast with the one from the band edge transition while passivation method can blue-shift the PL peak.

We explore the impact of antisolvent and additive on the photophysical properties and probe a better understanding of optical response of the perovskite materials. We hold the 
view that the performance improvement focused on material quality alone without complete understanding of the physics of the carrier-light interaction will not provide adequate solutions to existing problems.

\subsubsection{Light harvesting efficiency (LHE)}

The light harvesting efficiency (LHE) is the fraction of absorbed photons at a particular wavelength, it can be calculated from its absorbance using equation 1[11].

$$
\operatorname{LHE}(\lambda)=1-10^{-\mathrm{A}(\lambda)}
$$

where A connotes the oscillator strength of the sensitizer at a particular wavelength. Largely, a higher LHE value increases the photocurrent feedback and consequently the efficiency of the device. The LHE is a vital parameter for calculating the APCE (absorbed photon conversion efficiency) of a solar cell. This relies on the number of absorbed photons in the active layer of the cell at the excitation wavelength. The LHE value of $\mathrm{MAPbI}_{3}$ thin film calculated at its respective $\lambda_{\max }$ was 0.425 (42.5\%). Therefore, LHE is favourable for photovoltaic application.

\subsubsection{Absorption coefficient ( $\alpha)$}

The absorption coefficient of a semiconductor material at a specific wavelength indicates the area on which most of the light is absorbed. It describes how deep into a material light of specific wavelength can penetrate before it is absorbed. The absorption coefficient $(\alpha)$ can be evaluated using the Beer-Lambert's relation in equation 2 [12].

$$
\alpha=\frac{2.303 * A}{t}
$$

where A represents absorbance while $t$ stand for thickness of thin film

The absorption equivalent to the transfer of charge carriers from valence band to conduction band is employed to measure the band gap of the material. From the absorption, the value of absorption coefficient $\left(1.84 \times 10^{4} \mathrm{~cm}^{-1}\right)$ was calculated. The absorption of $\mathrm{CH}_{3} \mathrm{NH}_{3} \mathrm{PbI}_{3}$ film exhibit a sharp edge around the wavelength of $797.50 \mathrm{~nm}$, in close similarity to GaAs, CdTe, CIGS and c-Si [13]. Hence very thin absorber films are adequate for perovskite solar cells due to long exciton diffusion length unlike organic semiconductor with short exciton diffusion length.

\subsubsection{Optical band gap energy}

The optical energy gap that exist within the bands of any material relates to the absorption coefficient $(\alpha)$ and the incident photon energy (hv) according to the Tauc's relation in equation 3[14]:

$$
\alpha h v=A\left(h v-E_{g}\right)^{n}
$$

where $\mathrm{h}$ is the Planck's constant, $v$ represents frequency of incident photons, A is a constant, its value is based on the transition probability and at times known as the band tailing parameter, $\mathrm{n}$ is an index, and its value relies on the type of the electronic transition during the absorption process. The value of $n=1 / 2$ for direct transition and $n=2$ for indirect transition. In this case, $\mathrm{MAPI}_{3}$ is a semiconductor with a direct the band gap and suitable 
with $n=1 / 2 . E(h v)$ is the photon energy, $E_{g}$ is the optical energy gap, which located within the localized states around the mobility edges. The band gap was evaluated by plotting the $(\alpha h v)^{2}$ values versus $\mathrm{hv}(\mathrm{eV})$ as shown in the Figure 8 . The Tauc's region is extrapolated to $(\alpha h v)^{2}=0$ to obtain the band gap. We obtained a value of $1.55 \mathrm{eV}$.

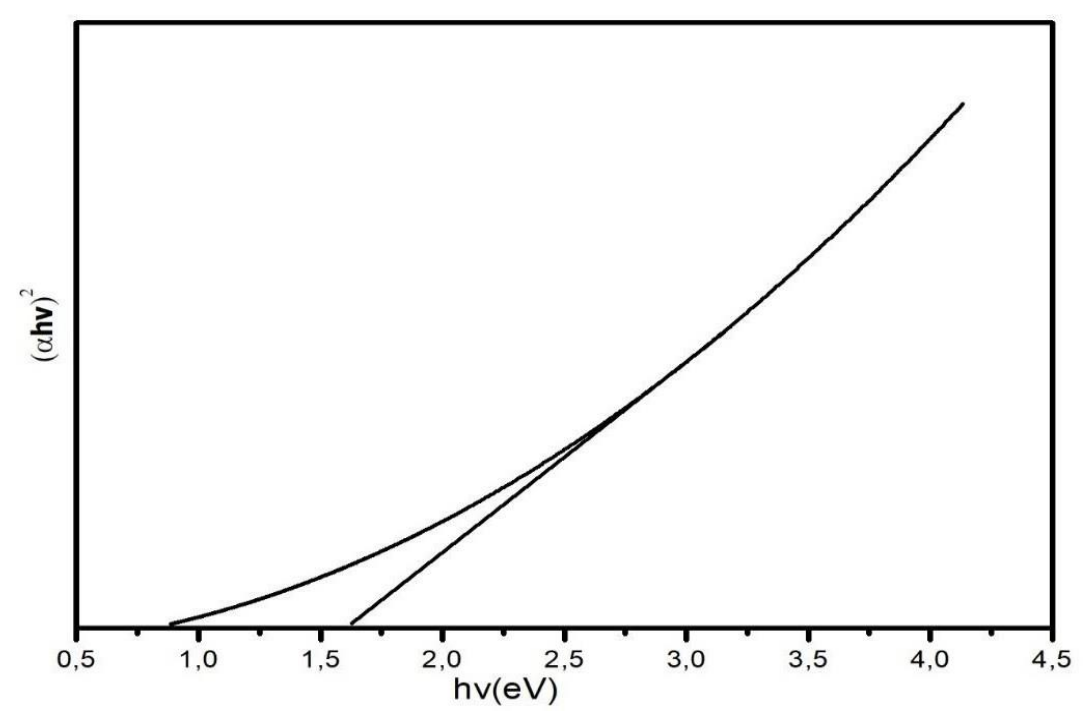

Figure 8. The optical band was calculated by using the Tauc plots of $(\alpha h v)^{2}$ versus E(eV).

\subsubsection{Extinction Coefficient (k)}

The extinction coefficient is the measurement of the small portion of light lost because of dispersion and absorption in a medium. It can be evaluated from the values of $\alpha$ and $\lambda$ using Equation 4 below ${ }^{15}$.

$$
k=\frac{\alpha \lambda}{4 \pi}
$$

The value of $\mathrm{k}$ was calculated to be 0.116 at $797.50 \mathrm{~nm}$. This is the value of $\mathrm{k}$ in the NIR region. At wavelengths over $600 \mathrm{~nm}$, the extinction coefficient $\mathrm{k}$ of the $\mathrm{CH}_{3} \mathrm{NH}_{3} \mathrm{PbI}_{3}$ perovskite takes value less than at shorter wavelengths and reduces down to near zero in the near-bandgap region [31]. Therefore, 0.116 is the value of the amount of light loss at that particular wavelength.

\subsubsection{Refractive Index (n) and Dielectric constant $(\varepsilon)$}

Refractive index and the extinction coefficient are fundamental quantities while examining the photonics aspect of optoelectronic devices, which jointly formulate the complex dielectric function. In solar cells, this parameter is key for comprehension and improving the cavity electron-optics of the device. 
Considering that light is an electromagnetic radiation, two quantities are primarily linked together. The refractive index is connected the extent to which the speed of light is diminished within a substance, while the dielectric constant shows us at what extent the electric field is diminished in a material. The refractive index was determined from Equation 5 below [16].

$$
n=\frac{(1+R)}{(1-R)}+\sqrt{\frac{4 R}{(1-R)^{2}}-K^{2}}
$$

The real (n) and imaginary $(\mathrm{k})$ parts of the refractive index are connected to dielectric constants with the real part $\left(\varepsilon_{\mathrm{r}}=\mathrm{n}^{2}-\mathrm{k}^{2}\right)$ and imaginary part $\left(\varepsilon_{\mathrm{i}}=2 \mathrm{nk}\right)$. Imaginary aspect of refractive index is correlated to the strength of absorption loss at a certain wavelength (extinction coefficient). The refractive index of $\mathrm{MAPbI}_{3}$ thin film was calculated to be 2.7 and the real dielectric constant is 4.81 which compare favourably with the values reported in the literature $[17,18]$. The $\mathrm{MAPbI}_{3}$ possesses a high dielectric value like its inorganic counterpart. In material optimisation the following affect the values of $\mathrm{n}$ and $\varepsilon$ : thickness of the layer, morphology, material composition, deposition method and material anisotropy.

\subsubsection{Optical conductivity $(\sigma)$}

The optical response of a material is mostly investigated with regards to its optical conductivity, which applies to the optical feedback of a material to photon energy and it depends directly on the absorption coefficient and the refractive index of the material. The optical conductivity was estimated using relation below [19].

$$
\sigma=\frac{\alpha n c}{4 \pi}
$$

here $\mathrm{c}$ is the speed of light, Our estimated value of the optical conductivity is $1.19 \mathrm{x}$ $10^{10} \mathrm{~s} / \mathrm{m}$.

\subsubsection{Anti-Stokes shift}

The rare case of an anti-Stokes shift luminescence, in which photons are emitted with larger energy (shorter wavelength) than absorbed is vividly seen as illustrated in the Figures 9. The above behaviour observed in Figure 6 confirms that $\mathrm{MAPbI}_{3}$ has nearly no defects or impurities, and the free carriers in the bands control the optical responses. It also verified that excitons do not appear to be predominant for $\mathrm{MAPbI}_{3}$, which agrees with absorption spectrum shown in Figure 6. Possible justification for this unusual behaviour is attributed to homogeneous broadening [24]. Suggestion was made that an effective electron-phonon interplay is accountable for the high anti-stokes PL[25]. This is termed anti-stoke shift mechanism and can be attributed to up-conversion in luminescent materials. It serves as an alternative approach for tackling energy loss by converting unused NIR photons to visible light photons. 


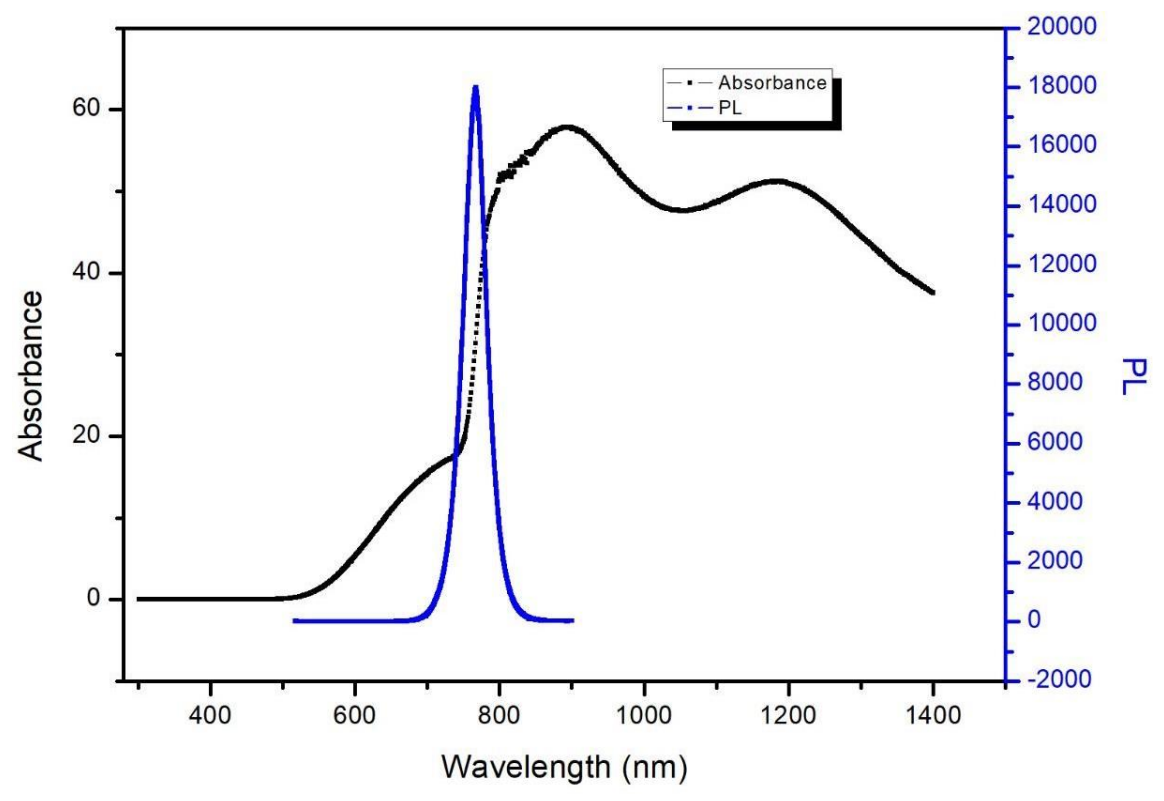

Figure 9. Absorption and PL properties of $\mathrm{MAPbI}_{3} \mathrm{SC}$

\subsection{Conclusion}

In summary we have effectively examined the impact of antisolvent and additive on the structural and morphological properties of the thin film, more importantly on the optical properties of halide perovskite. The results obtained relate to the sharp crystallinity of the perovskite material, which can be produced through a one-step technique with antisolvent and additive treatment. Contrary to suggested earlier techniques wherein crystallization was guided by employing high temperatures during precursor deposition, the significance of our strategy is the formation of perovskite thin film with low temperature which is needed for mass scale production. Also, the incorporation of DMSO has been instrumental in suppressing recombination process. The dielectric constant is at 4.8 for perovskite iodide. This implies that perovskite can store up charges as high-capacitance condensers [18]. Our measurements yielded values of band gap and optical conductivity of $1.55 \mathrm{eV}$ and $1.19 \mathrm{x}$ $10^{10} \mathrm{~s} / \mathrm{m}$, respectively. The real part of the refractive index was 2.7 which is considerably suitable for optical application. This also suggest that the $\mathrm{CH}_{3} \mathrm{NH}_{3} \mathrm{PbI}_{3}$-based solar cells can be useful to top cell in silicon-based tandem solar cells. The unusual anti-Stokes shift indicates an intense electron-phonon coupling in lead halide perovskites, which might be beneficial for certain applications such as up-conversion nanomaterials, Bio-imaging. All these parameters obtained are confirmations of the evidence that optical excitation in perovskite is generated by Wannier-Mott excitation [23], which indicate the creation of free charge carrier responsible for the photovoltaic effect.

Our findings give insight into the design of thin film optoelectronic devices. Additionally, the findings reveal another way of investigating the limited optical properties of the perovskite materials. Material optimization that leads to optical enhancement is the key before fabrication of photovoltaic device. Hence, we project that hybrid perovskite is the front runner in photovoltaic and optoelectronic applications as the material has superb optical and electronic properties that can revolutionize solar industry sector especially in 
this fourth industrial generation. We further propose that the performance improvement focused on material quality alone without complete understanding of the physics of the carrier-light interaction will not be beneficial to optoelectronic endeavours.

\section{ACKNOWLEDGEMENTS}

We acknowledge the support from the Council for Scientific and Industrial Research (CSIR) and financial support from the National Research Foundation (NRF), South Africa.

\section{References}

1. J. S. Manser, M. I. Saidaminov, J. A. Christians, O. M. Bakr, P. V. Kamat, Making and Breaking of Lead Halide Perovskites. Acc. Chem. Res. 49, 330-338. (2016)

2. Z. Song, S. C. Watthage, A. B. Phillips, M. J. Heben, Pathways Toward HighPerformance Perovskite Solar Cells: Review of Recent Advances in Organo-Metal Halide Perovskites for Photovoltaic Applications. J. Photonics Energy. 6, 022001. (2016)

3. H.D. Kim, H. Ohkita, H. Benten, S. Ito, Photovoltaic performance of perovskite solar cells with different grain size, Adv. Mater. 28 917-922. (2016)

4. Y.F. Wang, J. Wu, P. Zhang, D.T. Liu, T. Zhang, L. Ji, X.L. Gu, Z.D. Chen, S.B. $\mathrm{Li}$, Stitching triple cation perovskite by a mixed anti-solvent process for high performance perovskite solar cells, Nano Energy 39 616-625. (2017)

5. Y.F. Wang, S.B. Li, P. Zhang, D.T. Liu, X.L. Gu, H. Sarvari, Z.B. Ye, J. Wu, Z.M. Wang, Z.D. Chen, Solvent annealing of PbI2 for the high-quality crystallization of perovskite films for solar cells with efficiencies exceeding $18 \%$, Nanoscale 8 19654-19661. (2016)

6. T.M.Watson, J. Troughton, K. Hooper, Humidity resistant fabrication of $\mathrm{CH}_{3} \mathrm{NH}_{3} \mathrm{PbI} 3$ perovskite solar cells and modules, Nano Energy 39 60-68. (2017)

7. S. Sun, T. Salim, N. Mathews, M. Duchamp, C. Boothroyd, G. Xing, T. C. Sum, Y. M. Lam, The origin of high efficiency in low-temperature solution-processable bilayer organometal halide hybrid solar cells, Energy Environ. Sci. 7 399- 407. (2014)

8. F. Yang, M.A. Kamarudin, P. Zhang, G. Kapil, T. Ma, and S.Hayas. Enhanced Crystallization by Methanol Additive in Antisolvent for Achieving High-Quality MAPbI3 Perovskite Films in Humid Atmosphere. ChemSusChem 11 2348-235. (2018)

9. Y. Yang, K.H. Ri, A.Y. Mei, L.F. Liu, M. Hu, T.F. Liu, X. Li, H.W. Han, The size effect of $\mathrm{TiO}_{2}$ nanoparticles on a printable mesoscopic perovskite solar cell, J. Mater. Chem. A 3 9103-9107. (2015)

10. M.H. Lv, X. Dong, X. Fang, B.C. Lin, S. Zhang, X.Q. Xu, J.N. Ding, N.Y. Yuan, Improved photovoltaic performance in perovskite solar cells based on $\mathrm{CH} 3 \mathrm{NH} 3 \mathrm{PbI} 3$ films fabricated under controlled relative humidity, RSC Adv. 5 93957-93963.(2015) 
11. M. Pazoki, U. B. Cappel, E. M. J. Johansson, A. Hagfeldt and G. Boschloo. Characterization techniques for dye-sensitized solar cells. Energy Environ. Sci., 10 672. (2017)

12. J.I. Pankove. Optical processes in semiconductors (1st ed.), Prentice Hall, New Jersy pp 34. ISBN10 0136380239. (1971)

13. S.D. Wolf, J. Holovsky, S.J. Moon, P. Löper, B. Niesen, et al. organometallic halide perovskites: Sharp optical absorption edge and its relation to photovoltaic performance. J Phys Chem Lett 5 1035-1039.(2014)

14. J. Tauc, Amorphous and Liquid Semiconductors, Plenum, New York, (1974)

15. M. Yıldırım, F. Özel, N. Tuğluoğlu, Ö.F. Yüksel, M. Kuş. Optical characterization of Cu2ZnSnSe4-xSx nanocrystals thin film J. Alloy Compd., 666 pp. 144- 152. (2016)

16. M. Yıldırım, F. Özel, A. Sarılmaz, A. Aljabour, I. Patır. Investigation of structural, optical and dielectrical properties of Cu2WS4thin film J. Mater. Sci.: Mater. Electron. 289 pp. 6712-6721. (2017)

17. Nam-Gyu Park. Methodologies for high efficiency perovskite solar cells, Nano Convergence (2016).

18. M. Samiee, S. Konduri, B. Ganapathy, R. Kottokkaran, H.A. Abbas, A. Kitahara, P. Joshi, L. Zhang, M.Noack, V.Dalal, Defect density and dielectric constant in perovskite solar cells. Appl. Phys. Lett. 105153502 (2014)

19. M.M. Abd El-Raheem. Optical properties of GeSeTl thin films J. Phys.: Condens. Matter, 19216209 pp. 1-7. (2007)

20. W. Shockley and H. J. Queisser, Detailed Balance Limit of Efficiency of pn Junction Solar Cells J. Appl. Phys. 32510 (1961)

21. U. Rau and J. H. Werner, Radiative efficiency limits of solar cells with lateral band-gap fluctuations. Appl. Phys. Lett. 84 3735. (2004)

22. P. Nandakumar and C. Vijayan, Optical absorption and photoluminescence studies on CdS quantum dots in Nafion. Journal of applied physics 91, number 3. (2002)

23. N. Ashurov, B. L. Oksengendler, S. Maksimov, S. Rashiodva A. R. Ishteev , D. S. Saranin , I. N. Burmistrov, D. V. Kuznetsov , A. A. Zakhisov. Current state and perspectives for organo-halide perovskite solar cells. Part 1. Crystal structures and thin film formation, morphology, processing, degradation, stability improvement by carbon nanotubes. A review. Modern Electronic Materials 3 1-25. (2017)

24. C. Wehrenfennig, M. Liu, H. J. Snaith, M. B. Johnston, and L. M. Herz, Homogeneous Emission Line Broadening in the Organo Lead Halide Perovskite $\mathrm{CH}_{3} \mathrm{NH}_{3} \mathrm{PbI} 3-\mathrm{xCl}$ J. Phys. Chem. Lett. 5, 1300. (2014)

25. S.-T. Ha, C. Shen, J. Zhang, and Q. Xiong, Laser cooling of organic-inorganic lead halide perovskites Nat. Photonics $\quad \begin{array}{llll}10 & 115 & \text { (2016) }\end{array}$ 
26. Pao-Hsun Huang, Yeong-Her Wang, Jhong-Ciao Ke and Chien-Jung Huang. The Effect of Solvents on the Performance of $\mathrm{CH}_{3} \mathrm{NH} 3 \mathrm{PbI} 3$ Perovskite Solar Cells, Energies 10599 (2017)

27. K. Tanaka, T. Takahashi, T. Ban, T. Kondo, K. Uchida, N. Miura, Comparative Study on the Excitons in Lead-Halide-Based Perovskite-Type Crystals $\mathrm{CH} 3 \mathrm{NH}_{3} \mathrm{PbBr} 3 \mathrm{CH} 3 \mathrm{NH} 3 \mathrm{PbI} 3$. Solid State Commun. 127 619-623. (2003)

28. F. Guo, S. Qiu, J. Hu, H. Wang, B. Cai, J. Li, X. Yuan, X. Liu, K. Forberich, C. J. Brabec, and Y. Mai. A Generalized Crystallization Protocol for Scalable Deposition of High-Quality Perovskite Thin Films for Photovoltaic Applications, Adv. Sci. 6 1901067. (2019)

29. S. Tang, Y. H. Deng, X. P. Zheng, Y. Bai, Y. J. Fang, Q. F. Dong, H. T. Wei, J. S. Huang, Composition Engineering in Doctor-Blading of Perovskite Solar Cells Adv. Energy Mater. 7 1700302. (2017)

30. J. B. Li, R. Munir, Y. Y. Fan, T. Q. Niu, Y. C. Liu, Y. F. Zhong, Z. Yang, Y. S. Tian, B. Liu, J. Sun, D. M. Smilgies, S. Thoroddsen, A. Amassian, K. Zhao, S. Z. Liu, Phase Transition Control for High-Performance Blade-Coated Perovskite Solar Cells Joule 2 1313. (2018)

31. Hui Zhang and Johann Toudert. Optical management for efficiency enhancement in hybrid organic-inorganic lead halide perovskite solar cells, Science and Technology of advanced Materials, 19 no.1, 411-424. (2018)

32. T. Kinoshita, K. Nonomura, N. J. Jeon, F. Giordano, A. Abate, S. Uchida, T. Kubo, Sang Il Seok, M. K. Nazeeruddin, A. Hagfeldt, M. Gra"tzel and H. Segawa. Spectral splitting photovoltaics using perovskite and wideband dye-sensitized solar cells. Nature communications 68834 (2015) 